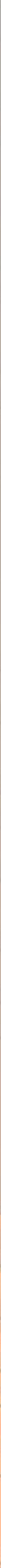




\title{
Finite dimensional Hopf actions on algebraic quantizations
}

\author{
Pavel Etingof and Chelsea Walton
}

Let $k$ be an algebraically closed field of characteristic zero. In joint work with J. Cuadra, we showed that a semisimple Hopf action on a Weyl algebra over a polynomial algebra $k\left[z_{1}, \ldots, z_{s}\right]$ factors through a group action, and this in fact holds for any finite dimensional Hopf action if $s=0$. We also generalized these results to finite dimensional Hopf actions on algebras of differential operators. In this work we establish similar results for Hopf actions on other algebraic quantizations of commutative domains. This includes universal enveloping algebras of finite dimensional Lie algebras, spherical symplectic reflection algebras, quantum Hamiltonian reductions of Weyl algebras (in particular, quantized quiver varieties), finite $W$-algebras and their central reductions, quantum polynomial algebras, twisted homogeneous coordinate rings of abelian varieties, and Sklyanin algebras. The generalization in the last three cases uses a result from algebraic number theory due to A. Perucca.

\section{Introduction}

Throughout this paper, $k$ will denote an algebraically closed field of characteristic zero. In [Etingof and Walton 2014, Theorem 1.3], we showed that any semisimple Hopf action on a commutative domain over $k$ factors through a group action. Likewise, it was established in our joint work with Juan Cuadra that the same conclusion holds for semisimple Hopf actions on Weyl algebras $\boldsymbol{A}_{n}\left(k\left[z_{1}, \ldots, z_{s}\right]\right)$ [Cuadra et al. 2015, Proposition 4.3]. Moreover, we showed that it holds for any (not necessarily semisimple) finite dimensional Hopf action on $\boldsymbol{A}_{n}(k)$ [Cuadra et al. 2016, Theorem 1.1], and, more generally, on algebras of differential operators of smooth affine varieties [Cuadra et al. 2016, Theorem 1.2]. Finally, in [Etingof and Walton 2016] we extended these results to certain finite dimensional Hopf actions on deformation quantizations (i.e., formal quantum deformations) of commutative domains. We say that there is no finite quantum symmetry in the settings above.

MSC2010: primary 16T05; secondary $16 \mathrm{~S} 80,13 \mathrm{~A} 35,16 \mathrm{~S} 38$.

Keywords: algebraic quantization, filtered deformation, Hopf algebra action, quantum polynomial algebra, Sklyanin algebra, twisted coordinate ring. 
The goal of this paper is to establish no finite quantum symmetry results for finite dimensional Hopf actions on other algebraic quantizations of commutative domains, i.e., quantizations whose parameters are elements of $k$ (rather than formal variables). We now summarize our main results for various classes of algebraic quantizations.

1A. Semisimple Hopf actions on filtered quantizations. Our first main result concerns Hopf actions on filtered deformations (or filtered quantizations) of commutative domains, that is, on filtered $k$-algebras $B$ where the associated graded algebra $\operatorname{gr}(B)$ is a commutative finitely generated domain.

Let $B$ be a $\mathbb{Z}_{+}$-filtered algebra over $k$ such that $\operatorname{gr}(B)$ is a commutative finitely generated domain. We will see that for sufficiently large primes $p$, the algebra $B$ admits a reduction $B_{p}$ modulo $p$, which is a domain over $\overline{\mathbb{F}}_{p}$. Namely, there exists an $R$-order $B_{R} \subset B$ over some finitely generated subring $R \subset k$, and

$$
B_{p}=B_{\psi, p}:=B_{R} \otimes_{R} \overline{\mathbb{F}}_{p}
$$

for a homomorphism $\psi: R \rightarrow \overline{\mathbb{F}}_{p}$. (For details on $R$-orders in $B$, see Section 2A below).

Recall that a ring $A$ is $P I$ if it satisfies a polynomial identity over $\mathbb{Z}$. By Posner's and Ore's theorems [Posner 1960; Ore 1931; McConnell and Robson 2001, Theorem 13.6.5 and Corollary 1.14], a domain $A$ is PI if and only if it is an Ore domain and its division ring of fractions $\operatorname{Frac}(A)$ is a central division algebra. In this case, $\operatorname{Frac}(A)$ is a division ring that is dimension $d^{2}$ over its center, where $d$ is the $P I$ degree of $A$ [McConnell and Robson 2001, Definition 13.6.7].

Definition 1.1. Given $B$ as above, we say that $B$ is an algebra with PI reductions if it admits an order $B_{R}$ such that $B_{p}$ is PI for sufficiently large $p$ (with any choice of $\psi)^{1}$

Theorem 2.4. If $B$ is an algebra with PI reductions, then any semisimple Hopf action on $B$ factors through a group action.

Note that when the Hopf action preserves the filtration of $B$, Theorem 2.4 (even without the PI reduction assumption) is proved in [Etingof and Walton 2014, Proposition 5.4]; our main achievement here is that we eliminate this requirement.

A basic example of an algebra with PI reductions is the Weyl algebra $B=\boldsymbol{A}_{n}(k)$, and, in fact, the proof of Theorem 2.4 is analogous to the proof of [Cuadra et al. 2015, Theorem 4.1], which addresses this case. Moreover, a wide range of filtered quantizations (each defined in Section 2B below) are algebras with PI reductions, resulting in the following corollary.

\footnotetext{
${ }^{1}$ It follows from Lemma 2.1(ii) below that if this condition holds for one pair $\left(R, B_{R}\right)$, then the condition holds for all such pairs.
} 
Corollary 2.6. Let $B$ be one of the following filtered $k$-algebras:

(i) any filtered quantization B generated in filtered degree one; in particular, the enveloping algebra $U(\mathfrak{g})$ of a finite dimensional Lie algebra $\mathfrak{g}$, or the algebra $D_{\omega}(X)$ of twisted differential operators on a smooth affine irreducible variety $X$;

(ii) a finite $W$-algebra or its quotient by a central character;

(iii) a quantum Hamiltonian reduction of a Weyl algebra by a reductive group action; in particular, the coordinate ring of a quantized quiver variety;

(iv) a spherical symplectic reflection algebra; or

(v) the tensor product of any of the algebras above with any commutative finitely generated domain over $k$.

Then any semisimple Hopf action on $B$ factors through a group action.

Other applications of Theorem 2.4 have been investigated recently by Lomp and Pansera [2015]; for instance, they establish no finite semisimple quantum symmetry on certain iterated differential operator rings.

Remark 1.2. We do not know if a filtered quantization of a finitely generated commutative domain over $k$ must be an algebra with PI reductions (i.e., if the PI reduction assumption is, in fact, vacuous); see the question in [Cuadra et al. 2015, Introduction] and [Etingof 2016, Question 1.1]. This is of independent interest in noncommutative ring theory.

1B. Finite dimensional Hopf actions on filtered quantizations. Like [Cuadra et al. 2015, Theorem 4.2], Theorem 2.4 and hence Corollary 2.6 hold for Hopf-Galois actions of any (not necessarily semisimple) finite dimensional Hopf algebra (see Theorem 3.1). The proof is parallel to the proofs of Theorem 2.4 and [Cuadra et al. 2015, Theorem 4.2].

Moreover, it turns out that even without the Hopf-Galois assumption, Theorem 2.4 extends to nonsemisimple Hopf actions for a somewhat more restrictive class of quantizations. To see this, let us recall some algebras introduced in [Cuadra et al. 2016]. Notation $1.3\left(B, B_{p^{m}}, C_{m}, D_{p^{m}}, Z, Z(m)\right)$. Let $B$ be a quantization with PI reductions, and let $B_{p^{m}}$ be the reduction of $B$ modulo $p^{m}$. Let $C_{m}$ be the center of $B_{p^{m}}, \operatorname{Frac}\left(C_{m}\right)$ be its ring of fractions, and $D_{p^{m}}:=B_{p^{m}} \otimes_{C_{m}} \operatorname{Frac}\left(C_{m}\right)$. The PI reduction condition implies that $D_{p^{m}}$ is the full localization (i.e., ring of fractions) of $B_{p^{m}}$. These algebras are defined over the truncated Witt ring $W_{m, p}$ of $\overline{\mathbb{F}}_{p}$; cf. [Cuadra et al. 2016, Sections 2.1, 2.3, 2.4]. Let $Z$ be the center of the central division algebra $D_{p}$. (Here and below, to lighten the notation, we often suppress dependence on $p$.) Let $Z_{m}$ be the center of $D_{p^{m}}$, and let $Z(m)$ be its image in $D_{p}$ under the map $D_{p^{m}} \rightarrow D_{p}$ (so $Z(1)=Z$ ). It is easy to see that $Z(m) \subset Z(m-1)$. 
Definition 1.4. We say that an algebra $B$ with PI reductions is nondegenerate if for almost all $p$ one has $\bigcap_{m \geq 1} Z(m)=\overline{\mathbb{F}}_{p}$.

Theorem 3.5. If $B$ is a nondegenerate algebra with PI reductions, then any finite dimensional Hopf action over $B$ factors through a group action (i.e., the condition that $H$ is semisimple in Theorem 2.4 can be dropped).

The proof of Theorem 3.5 is similar to the proof of [Cuadra et al. 2016, Theorem 1.2].

To illustrate when the nondegeneracy condition holds, recall that $\operatorname{gr}(B)$ carries a natural Poisson bracket. Namely, if $B$ is commutative, this bracket is zero; otherwise, if $d$ is the largest integer such that $\left[F_{i} B, F_{j} B\right] \subset F_{i+j-d} B$, then for $a_{0}$ $\in \operatorname{gr}_{i}(B)$ and $b_{0} \in \operatorname{gr}_{j}(B)$, the Poisson bracket $\left\{a_{0}, b_{0}\right\}$ is the projection of $[a, b]$ to $\operatorname{gr}_{i+j-d}(B)$, where $a \in F_{i} B$ and $b \in F_{j} B$ are any lifts of $a_{0}$ and $b_{0}$, respectively. Thus, $\operatorname{gr}(B)=O(X)$, where $X$ is an irreducible Poisson algebraic variety.

The nondegeneracy assumption is satisfied, in particular, when $X$ is a generically symplectic Poisson variety, i.e., one having a symplectic dense open subset; see Theorem 3.6. Therefore, Theorem 3.5 holds for many of the examples of Corollary 2.6- quantum Hamiltonian reductions of Weyl algebras, central reductions of finite $W$-algebras, spherical symplectic reflection algebras, and tensor products thereof (see Corollary 3.7).

1C. Quantum polynomial algebras. For our next main result, we consider finite dimensional Hopf actions on quantum polynomial algebras (or quantized coordinate rings of affine $n$-space):

$$
k_{q}\left[x_{1}, \ldots, x_{n}\right]:=k\left\langle x_{1}, \ldots, x_{n}\right\rangle /\left(x_{i} x_{j}-q_{i j} x_{j} x_{i}\right),
$$

where $\boldsymbol{q}=\left(q_{i j}\right), q_{i j} \in k^{\times}$with $q_{i i}=1$ and $q_{i j} q_{j i}=1$. Thus we can view $\boldsymbol{q}$ as a point of the algebraic torus $\left(k^{\times}\right)^{n(n-1) / 2}$ with coordinates $q_{i j}$ for $i<j$.

There are many examples of semisimple Hopf actions on $k_{q}\left[x_{1}, \ldots, x_{n}\right]$ that do not factor through group actions; the parameters $q_{i j}$ are roots of unity in these examples. See, for instance, [Chan et al. 2016, Theorem 0.4; Etingof and Walton 2014, Example 5.10; Kirkman et al. 2009, Examples 7.4-7.6]. Still, we establish the following result.

Let $\langle\boldsymbol{q}\rangle$ be the subgroup in $\left(k^{\times}\right)^{n(n-1) / 2}$ generated by $\boldsymbol{q}$, and let $G_{\boldsymbol{q}}$ be its Zariski closure. Let $G_{\boldsymbol{q}}^{0}$ be the connected component of the identity in $G_{\boldsymbol{q}}$.

Theorem 1.5 (Theorem 4.1). Let $H$ be a semisimple Hopf algebra of dimension d. If the order of $G_{q} / G_{q}^{0}$ is coprime to $d !$, then any $H$-action on $B:=k_{q}\left[x_{1}, \ldots, x_{n}\right]$ factors through a group action.

If each $q_{i j}$ is a root of unity of order $r_{i j}$, then $\left|G_{\boldsymbol{q}} / G_{\boldsymbol{q}}^{0}\right|=\operatorname{lcm}\left\{r_{i j}\right\}_{i<j}$. In particular, if $n=2$, i.e., if $B=k\langle x, y\rangle /(x y-q y x)$, then the condition on $\boldsymbol{q}=q \in k^{\times}$in 
Theorem 1.5 is that the order of $q$ is coprime to $d$ ! or infinite. On the other hand, the condition on $\boldsymbol{q}$ in Theorem 1.5 is also satisfied if each $q_{i j}$ is not a root of unity and the set of the $q_{i j}$ is multiplicatively independent; here, $\left|G_{\boldsymbol{q}} / G_{\boldsymbol{q}}^{0}\right|=1$. See Example A.3 for a discussion of how to compute $\left|G_{q} / G_{q}^{0}\right|$ in general.

One may compare Theorem 1.5 to a similar result, Theorem 4.3 of [Chan et al. 2014], in the case where the Hopf action preserves the grading of $k_{q}\left[x_{1}, \ldots, x_{n}\right]$. But note that without the degree-preserving assumption, semisimplicity is still needed in Theorem 1.5; see [Etingof and Walton 2015; 2016, Example 3.6] for counterexamples for $n=1,3$, respectively.

Moreover, Theorem 1.5 is valid for finite dimensional Hopf algebras in the HopfGalois case, where we can replace the condition "coprime to $d$ !" with "coprime to $d$ " (Proposition 5.1). Also, Theorem 1.5 has a straightforward generalization (with the same proof) to actions on the quantum torus $k_{q}\left[x_{1}^{ \pm 1}, \ldots, x_{n}^{ \pm 1}\right]$.

Another generalization of Theorem 1.5 to the nonsemisimple case can be made under a nondegeneracy assumption. Recall that $\boldsymbol{q}$ may be viewed as a skewsymmetric bicharacter on $\mathbb{Z}^{n}$ with values in $k^{\times}$, with $\boldsymbol{q}\left(e_{i}, e_{j}\right)=q_{i j}$ for the standard basis $\left\{e_{i}\right\}$. A bicharacter $\boldsymbol{q}$ is called nondegenerate if the character $\boldsymbol{q}(a, \cdot): \mathbb{Z}^{n} \rightarrow k^{\times}$ is nontrivial whenever $a \neq 0$. Note that unlike skew-symmetric bilinear forms (which are always degenerate in odd dimensions), a skew-symmetric bicharacter can be nondegenerate for any $n \geq 2$.

Theorem 5.2. Let $H$ be a finite dimensional Hopf algebra of dimension d acting on $B:=k_{\boldsymbol{q}}\left[x_{1}, \ldots, x_{n}\right]$. Assume that the order of $G_{\boldsymbol{q}} / G_{\boldsymbol{q}}^{0}$ is coprime to $d !$, and $\boldsymbol{q}$ is a nondegenerate bicharacter. Then, the action of $H$ on $B$ factors through a group algebra.

It is shown in Example 5.5 that Theorems 1.5 and 5.2 fail when hypotheses are removed; these examples involve actions of the nonsemisimple 4-dimensional Sweedler Hopf algebra.

\section{D. Twisted homogeneous coordinate rings of abelian varieties and Sklyanin}

algebras. Let $X$ be an abelian variety over $k$, let $\mathcal{L}$ be an ample line bundle on $X$, and let $\sigma: X \rightarrow X$ be an automorphism given by translation by a point $s \in X$. Then we can define the twisted homogeneous coordinate ring

$$
B(X, \sigma, \mathcal{L}):=\bigoplus_{n=0}^{\infty} H^{0}\left(X, \bigotimes_{i=0}^{n-1}\left(\sigma^{i}\right)^{*} \mathcal{L}\right),
$$

with twisted multiplication $f * g:=f\left(\sigma^{n}\right)^{*}(g)$, where $f$ is of degree $n$ [Artin and Van den Bergh 1990]. It is well-known that $B(X, \sigma, \mathcal{L})$ is a domain, and if $|\sigma|<\infty$, then $B(X, \sigma, \mathcal{L})$ is a PI domain of PI degree $|\sigma|$.

Let $G_{\sigma}$ be the Zariski closure of the subgroup $\left\{s^{i}\right\}_{i \in \mathbb{Z}}$, and let $G_{\sigma}^{0}$ be the connected component of the identity in $G_{\sigma}$. 
Theorem 1.6 (Theorem 6.1). If $H$ is a semisimple Hopf algebra of dimension d, and if the order of $G_{\sigma} / G_{\sigma}^{0}$ is coprime to $d !$, then any $H$-action on $B(X, \sigma, \mathcal{L})$ factors through a group action.

In particular, if the subgroup $\left\{s^{i}\right\}_{i \in \mathbb{Z}}$ is Zariski-dense in $X$, then any semisimple Hopf action on $B(X, \sigma, \mathcal{L})$ factors through a group action. Moreover, if $X=: E$ is an elliptic curve, the condition on $\sigma$ in Theorem 6.1 is that the order of $\sigma$ is coprime to $d$ ! or infinite.

Lastly, we study semisimple Hopf actions on another class of quantizations: the 3-dimensional Sklyanin algebras $S(a, b, c)$ (Definition 6.3). To $S(a, b, c)$, one can naturally associate an elliptic curve $E_{a b c} \subset \mathbb{P}_{k}^{2}$ and an automorphism $\sigma_{a b c}$ given by translation by a point; see [Artin et al. 1990, Introduction].

Theorem 1.7. If $H$ is a semisimple Hopf algebra of dimension $d$, and if the order of $\sigma_{a b c}$ is coprime to $d !$ or infinite, then any $H$-action on $S(a, b, c)$ factors through a group action.

Remark 1.8. We believe that by adapting the techniques in this work, one could establish a version of Theorem 1.7 for semisimple Hopf actions on other elliptic algebras, such as in [Sklyanin 1982] (or, see [Smith and Stafford 1992]) and in [Etingof and Ginzburg 2010; Odesskiu 2002; Stephenson 1997]. Further, we believe that under an appropriate nondegeneracy condition, there are no finite quantum symmetry results for such elliptic algebras and for twisted homogeneous coordinate rings $B(X, \sigma, \mathcal{L})$ as above; compare to Theorem 5.2.

Our paper is organized as follows. We discuss semisimple Hopf actions on filtered quantizations in Section 2, nonsemisimple Hopf actions on filtered quantizations in Section 3, semisimple Hopf actions on quantum polynomial algebras in Section 4, nonsemisimple Hopf actions on quantum polynomial algebras in Section 5, and Hopf actions on twisted homogeneous coordinate rings of abelian varieties and Sklyanin algebras in Section 6. The results of Sections 4-6 rely on a number-theoretic result of Antonella Perucca discussed in the Appendix.

The notation and terminology of the introduction is used throughout this work, often without mention.

\section{Semisimple Hopf actions on filtered quantizations}

\section{A. The result on semisimple Hopf actions on quantizations with PI reductions.} In this section, we study actions of semisimple Hopf algebras $H$ on filtered quantizations $B$. Throughout this section, we let $B$ denote a $\mathbb{Z}_{+}$-filtered algebra over $k$ such that $\operatorname{gr}(B)$ is a commutative finitely generated domain; such $B$ will be referred to as a filtered quantization. 
Our goal is to prove Theorem 2.4. This result was established in [Cuadra et al. 2015] for $B$ a Weyl algebra, and we generalize those techniques for our purpose here.

Let $R$ be a finitely generated subring of $k$. By an $R$-order in a filtered quantization $B$ we mean an $R$-subalgebra $B_{R}$ of $B$ such that $\operatorname{gr}\left(B_{R}\right)$ is a finitely generated $R$-algebra which is projective as an $R$-module, and such that the natural map $\operatorname{gr}\left(B_{R}\right) \otimes_{R} k \rightarrow \operatorname{gr}(B)$ is an isomorphism of graded $k$-algebras.

Lemma 2.1. (i) Any filtered quantization $B$ admits an $R$-order $B_{R}$ for a suitable ring $R$.

(ii) For any two orders $B_{R}$ over $R$ and $B_{R^{\prime}}$ over $R^{\prime}$, there exists a finitely generated ring $R^{\prime \prime} \subset k$ containing $R$ and $R^{\prime}$, and admitting an $R^{\prime \prime}$-algebra isomorphism $\phi: B_{R} \otimes_{R} R^{\prime \prime} \rightarrow B_{R^{\prime}} \otimes_{R^{\prime}} R^{\prime \prime}$ such that $\mathrm{gr} \phi$ is an isomorphism.

Proof. (i) By the Hilbert basis theorem, the algebra $\operatorname{gr}(B)$ is finitely presented. This implies that so is $B$, as we can lift the generators and defining relations $\operatorname{of} \operatorname{gr}(B)$ to those of $B$.

More specifically, pick homogeneous generators $\bar{b}_{1}, \ldots, \bar{b}_{n}$ of $\operatorname{gr}(B)$ of degrees $m_{1}, \ldots, m_{n}$. Let

$$
p_{s}\left(\bar{b}_{1}, \ldots, \bar{b}_{n}\right)=0, \quad s=1, \ldots, r
$$

be a set of defining relations for $\operatorname{gr}(B)$, with $p_{s} \in k\left[X_{1}, \ldots, X_{n}\right]$ being homogeneous of degree $d_{s}$ (this set may be chosen to be finite by the Hilbert basis theorem). Let $b_{j}$ be lifts of $\bar{b}_{j}$ to $B$, and $\tilde{p}_{s}$ be homogeneous lifts of $p_{s}$ to $k\left\langle X_{1}, \ldots, X_{n}\right\rangle$. Then $\left[b_{i}, b_{j}\right]=f_{i j}\left(b_{1}, \ldots, b_{n}\right)$, where $f_{i j} \in k\left\langle X_{1}, \ldots, X_{n}\right\rangle$ is a noncommutative polynomial of filtration degree at most $m_{i}+m_{j}-1$, and $\tilde{p}_{s}\left(b_{1}, \ldots, b_{n}\right)=p_{s}^{\prime}\left(b_{1}, \ldots, b_{n}\right)$, where $p_{s}^{\prime} \in k\left\langle X_{1}, \ldots, X_{n}\right\rangle$ is a noncommutative polynomial of filtration degree at most $d_{s}-1$.

Let $g_{s}:=\tilde{p}_{s}-p_{s}^{\prime} \in k\left\langle X_{1}, \ldots, X_{n}\right\rangle$. Thus, we have relations

$$
\left[b_{i}, b_{j}\right]=f_{i j}\left(b_{1}, \ldots, b_{n}\right) \quad \text { and } \quad g_{s}\left(b_{1}, \ldots, b_{n}\right)=0
$$

in $B$. It is easy to see that these relations are defining, since they are already defining at the graded level.

Using relations (1), we can find a suitable finitely generated subring $R \subset k$ and define $B_{R} \subset B$ as follows. We take $\widetilde{R}$ to be the ring generated by all the coefficients of the polynomials $f_{i j}, g_{s}$, and set $R=\widetilde{R}[1 / f]$ for a suitable $f \in \widetilde{R}$. Now let $B_{R}$ be the subalgebra of $B$ generated over $R$ by $b_{1}, \ldots, b_{n}$. We can choose $f$ so that (1) are defining relations for $B_{R}$, and so that $B_{R}$ is an $R$-order on $B$, since for a suitable choice of $f, \operatorname{gr}\left(B_{R}\right)$ is a projective (in fact, free) $R$-module by Grothendieck's generic freeness lemma [Eisenbud 1995, Theorem 14.4]. This proves (i). 
(ii) Note that we have a natural isomorphism of filtered algebras

$$
\widetilde{\phi}: B_{R} \otimes_{R} k \rightarrow B_{R^{\prime}} \otimes_{R^{\prime}} k
$$

(as both are equal to $B$ ). This isomorphism is defined over some finitely generated ring $R^{\prime \prime} \subset k$ containing $R$ and $R^{\prime}$, which implies (ii).

Lemma 2.2. Suppose that $B$ is a filtered quantization that carries an action of a finite dimensional Hopf algebra $H$. Let $S$ be a finitely generated subring of $k$, and $B_{S}$ be an $S$-order in $B$. Then one can find a finitely generated subring $R \subset k$ containing $S$ and a Hopf order $H_{R} \subset H$ (cf. [Cuadra et al. 2015, Definition 2.1]), so that there is an induced action of $H_{R}$ on $B_{R}:=B_{S} \otimes_{S} R$ which gives the action of $H$ on $B$ upon tensoring over $R$ with $k$.

Proof. We use the method of [Cuadra et al. 2015, Section 2]. Pick homogeneous generators $\bar{b}_{1}, \ldots, \bar{b}_{n}$ of $\operatorname{gr}\left(B_{S}\right)$, and let $b_{j}$ be lifts of $\bar{b}_{j}$ to $B_{S}$. Choose a basis $\left\{h_{m}\right\}$ of $H$. We have

$$
h_{m} \cdot b_{j}=q_{m j}\left(b_{1}, \ldots, b_{n}\right)
$$

for some noncommutative polynomials $q_{m j} \in k\left\langle X_{1}, \ldots, X_{n}\right\rangle$. Let $R$ be generated over $S$ by the structure constants of $H$ in the basis $\left\{h_{m}\right\}$ and the coefficients of $q_{m j}$. Let $H_{R} \subset H$ be the span of $h_{m}$ over $R$. Then, $H_{R} \subset H$ is a Hopf order, and $H_{R}$ acts on $B_{R}$ by formula (2). The lemma is proved.

Thus, any action of $H$ on $B$ admits an $R$-order for some finitely generated ring $R \subset k$. Moreover, it is easy to see from Lemma 2.1(ii) that any two such orders over rings $R$ and $R^{\prime}$ can be identified after tensoring with some finitely generated ring $R^{\prime \prime} \subset k$ containing $R$ and $R^{\prime}$, so an order is essentially unique.

Now fix a ring $R$ and an $R$-order $B_{R} \subset B$ with an action of $H_{R}$. Let $p$ be a sufficiently large prime, and $\psi: R \rightarrow \overline{\mathbb{F}}_{p}$ be a character. Following [Cuadra et al. 2015, Section 2], let $H_{p}:=H_{R} \otimes_{R} \overline{\mathbb{F}}_{p}, B_{p}:=B_{R} \otimes_{R} \overline{\mathbb{F}}_{p}$ be the corresponding reductions of $H, B$ modulo $p$, where $\overline{\mathbb{F}}_{p}$ is an $R$-module via $\psi$. Then, $H_{p}$ acts on $B_{p}$ (by applying $\psi$ to the action of $H_{R}$ on $B_{R}$ ).

Lemma 2.3. For a sufficiently large prime $p, \operatorname{gr}\left(B_{p}\right)$, and hence $B_{p}$, is a domain.

Proof. We have $\operatorname{gr}\left(B_{p}\right)=\operatorname{gr}\left(B_{R}\right) \otimes_{R} \overline{\mathbb{F}}_{p}$. Therefore, the statement follows from [Grothendieck 1966, 9.7.7(i)] ("geometric irreducibility").

Theorem 2.4. If $B$ is an algebra with PI reductions, then any semisimple Hopf action on $B$ factors through a group action.

Proof. We may assume without loss of generality that the action of $H$ on $B$ is inner faithful (otherwise we can pass to an action of a quotient Hopf algebra).

Take $p \gg 0$. Then by [Cuadra et al. 2015, Proposition 2.4] (which applies with the same proof in our more general situation), $H_{p}$ acts inner faithfully on $B_{p}$. 
Moreover, as in [Cuadra et al. 2015, Lemma 2.5], $H_{p}$ is a semisimple cosemisimple Hopf algebra over $\overline{\mathbb{F}}_{p}$.

Since $B$ is an algebra with PI reductions, by Lemma 2.3, the algebra $B_{p}$ is a PI domain. Let $D_{p}$ be the division algebra of quotients of $B_{p}$. Then by [Etingof 2016, Corollary 3.2(ii)], $D_{p}$ is a central division algebra of degree $p^{n}$ for some $n \geq 0$ (which may depend on $p$ ). Moreover, $H_{p}$ acts inner faithfully on $D_{p}$ by [Skryabin and Van Oystaeyen 2006, Theorem 2.2].

Since $\operatorname{deg} D_{p}=p^{n}$ is coprime to $(\operatorname{dim} H)$ !, [Cuadra et al. 2015, Proposition 3.3(ii)] implies that $H_{p}$ is cocommutative. Thus, $H$ is cocommutative (as in the proof of [Cuadra et al. 2015, Theorem 4.1]), and thus is a group algebra.

2B. Some examples of filtered quantizations. As a consequence, Theorem 2.4 applies to semisimple Hopf actions on many classes of filtered quantizations. Namely, we will consider the following examples.

Twisted differential operators. Let $X$ be a smooth affine irreducible algebraic variety over $k$, and $\omega$ a closed 2-form on $X$. Then we define the algebra of twisted differential operators $D_{\omega}(X)$ to be the algebra generated by $O(X)$ and elements $L_{v}$ attached $k$-linearly to vector fields $v \in \operatorname{Der} O(X)$ on $X$, with defining relations

$$
L_{f v}=f L_{v}, \quad\left[L_{v}, f\right]=v(f), \quad\left[L_{v}, L_{w}\right]=L_{[v, w]}+\omega(v, w)
$$

for $f \in O(X), v, w \in \operatorname{Der} O(X)$. Then $D_{\omega}(X)$ carries a filtration defined by $\operatorname{deg} O(X)=0$ and $\operatorname{deg} L_{v}=1$ for $v \in \operatorname{Der} O(X)$, and $\operatorname{gr}\left(D_{\omega}(X)\right)=O\left(T^{*} X\right)$, the algebra of functions on the symplectic variety $T^{*} X$.

The filtered algebra $D_{\omega}(X)$ depends only on the cohomology class $[\omega]$ of $\omega$, and if $[\omega]=0$, then $D_{\omega}(X)=D(X)$, the algebra of usual differential operators on $X$. For more on twisted differential operators, see, e.g., [Bělinson and Bernstein 1993, Section 2].

Quantum Hamiltonian reductions. Let $G$ be a reductive algebraic group over $k$ with Lie algebra $\mathfrak{g}$, and let $(V,(\cdot, \cdot))$ be a faithful finite dimensional symplectic representation of $G$. Let $\boldsymbol{A}(V)$ be the Weyl algebra of $V$, generated by $v \in V$ with relations $[v, w]=(v, w)$ for $v, w \in V$. We have a natural action of $G$ on $\boldsymbol{A}(V)$ which preserves its filtration. In this case, we have a natural $G$-equivariant Lie algebra map $\hat{\mu}: \mathfrak{g} \rightarrow \boldsymbol{A}(V)$ called the quantum moment map, which quantizes the classical moment map $\mu: V \rightarrow \mathfrak{g}^{*}$, where $\mu(v)(a)=\frac{1}{2}(v, a v)$ for $v \in V, a \in \mathfrak{g}$. Now, given a character $\chi: \mathfrak{g} \rightarrow k$, we can define the algebra

$$
B(\chi):=[\boldsymbol{A}(V) / \boldsymbol{A}(V)(\hat{\mu}(a)-\chi(a), a \in \mathfrak{g})]^{G},
$$

called the quantum Hamiltonian reduction of $\boldsymbol{A}(V)$ by $G$ using $\chi$. It inherits a filtration from the Weyl algebra. See [Etingof 2007, Chapter 4] for further details. 
Assume that the moment map $\mu$ is flat, and that the scheme $\mu^{-1}(0)$ is reduced and irreducible (i.e., $\mu^{-1}(0)$ is a reduced irreducible complete intersection). In this case, $X:=\mu^{-1}(0) / G$ is an irreducible generically symplectic Poisson variety, and $B(\chi)$ is a filtered quantization of $O(X)$. See [Losev and Etingof 2015, Section 2.3; Nakajima 2015, Section 2(i)], and references therein for more details.

An interesting special case of this is when

$$
G=\left(\prod_{i=1}^{r} \mathrm{GL}\left(V_{i}\right)\right) / k^{\times} \quad \text { and } \quad V=\bigoplus_{i, j}\left(V_{i} \otimes V_{j}^{*}\right)^{\oplus m_{i j}},
$$

where $V_{i}$ are finite dimensional vector spaces and $m_{i j}=m_{j i}$ are positive integers with $m_{i i}$ even; i.e., $V$ is the space of representations of a doubled quiver, and $G$ is the group of linear transformations for this quiver. In this case, $B(\chi)$ is the quantized quiver variety; see, e.g., [Braden et al. 2012, Section 3.4]. The conditions under which $\mu$ is flat and $\mu^{-1}(0)$ is reduced and irreducible are given in [Crawley-Boevey 2001, Theorems 1.1 and 1.2].

Finite $W$-algebras. Let $\mathfrak{g}$ be a simple Lie algebra over $k$, and $e \in \mathfrak{g}$ a nilpotent element. To this data one can attach a Lie subalgebra $\mathfrak{m} \subset \mathfrak{g}$ with a character $\chi$, and a finite $W$-algebra is

$$
U(\mathfrak{g}, e):=(U(\mathfrak{g}) / U(\mathfrak{g})(a-\chi(a), a \in \mathfrak{m}))^{\mathrm{ad} \mathfrak{m}},
$$

a quantum Hamiltonian reduction of $U(\mathfrak{g})$. The algebra $U(\mathfrak{g}, e)$ has a filtration induced by the filtration in $U(\mathfrak{g})$, and its associated graded algebra is a polynomial algebra (of functions on the corresponding Slodowy slice). We refer the reader to [Losev 2010, Sections 2.3 and 2.4] and the references therein for details.

Also, the center $U(\mathfrak{g})^{\mathfrak{g}}$ of $U(\mathfrak{g})$ embeds into $U(\mathfrak{g}, e)$, so for any central character $\theta: U(\mathfrak{g})^{\mathfrak{g}} \rightarrow k$, one can consider the central reduction

$$
U_{\theta}(\mathfrak{g}, e):=U(\mathfrak{g}, e) /\left(a-\theta(a), a \in U(\mathfrak{g})^{\mathfrak{g}}\right) .
$$

Then $\operatorname{gr}\left(U_{\theta}(\mathfrak{g}, e)\right)=O(X)$, where $X$ is the nilpotent Slodowy slice, a generically symplectic Poisson variety.

Symplectic reflection algebras. Let $G$ be a finite group and $V$ a faithful finite dimensional symplectic representation of $G$, and assume that $V$ is not a direct sum of two nonzero symplectic representations. The symplectic reflection algebra $H_{t, c}(G, V)$ is the most general filtered deformation of $k G \ltimes S V$, where $\left[F_{i}, F_{j}\right] \subset F_{i+j-2}$; here $t \in k$, and $c$ is a conjugation invariant function on the set of symplectic reflections in $G$; see [Etingof 2007, Chapter 8].

Let $\boldsymbol{e}=|G|^{-1} \sum_{g \in G} g$ be the symmetrizing idempotent for $G$. Then, the algebra $\boldsymbol{e} H_{t, c}(G, V) \boldsymbol{e}$ is called the spherical symplectic reflection algebra. For $t=1$, it is a 
filtered quantization of $O(X)$, where $X=V / G$, a generically symplectic Poisson variety.

Remark 2.5. There are many other interesting examples of filtered quantizations, and our results given below can be extended to such examples. Since this extension is rather routine, we leave it outside the scope of this paper.

\section{C. Results on semisimple Hopf actions on specific filtered quantizations. Here} are some concrete applications of Theorem 2.4.

Corollary 2.6. Let $B$ be one of the following filtered $k$-algebras:

(i) any filtered quantization $B$ generated in filtered degree one; in particular, the enveloping algebra $U(\mathfrak{g})$ of a finite dimensional Lie algebra $\mathfrak{g}$, or the algebra $D_{\omega}(X)$ of twisted differential operators on a smooth affine irreducible variety $X$;

(ii) a finite $W$-algebra or its quotient by a central character;

(iii) a quantum Hamiltonian reduction of a Weyl algebra by a reductive group action; in particular, the coordinate ring of a quantized quiver variety;

(iv) a spherical symplectic reflection algebra; or

(v) the tensor product of any of the algebras above with any commutative finitely generated domain over $k$.

Then any semisimple Hopf action on B factors through a group action.

Note that in some of these cases, a stronger statement is true: any finite dimensional (not necessarily semisimple) Hopf action on $B$ factors through a group action; see Corollary 3.7 below. However, we still prefer to prove the weaker version here, since the proof is simpler (e.g., it does not require reduction modulo prime powers).

Proof. By Theorem 2.4, our job is to show that $B$ is an algebra with PI reductions. In other words, we need to show that the division algebra $D_{p}$ of fractions of $B_{p}$ is central (i.e., there is a " $p$-center") for $p \gg 0$. We do so below in each case.

(i) We show that if a filtered quantization $A$ of a commutative finitely generated domain $A_{0}$ over a field $F$ of characteristic $p>0$ is generated in degree one, then it is module-finite over its center after localization; this implies the required statement.

Let $A_{0}[i]$ be the degree $i$ part of $A_{0}$. Then $A_{0}[0]=A[0]$ is a finitely generated commutative domain. Let $\bar{a}_{1}, \ldots, \bar{a}_{n}$ be generators of $A_{0}[1]$ as an $A_{0}[0]$-module. Let $a_{i}$ be lifts of $\bar{a}_{i}$ to $A$. Then, $a_{i}$ and $A[0]$ generate $A$ as an algebra. Also, the operators $\left[a_{i}, \cdot\right]$ are derivations of $A[0]$, and hence vanish on $A[0]^{p}$. Thus, $A[0]^{p}$ is central in $A$. Let $K$ be the field of quotients of $A[0]^{p}$, and let $A^{\prime}:=A \otimes_{A[0]^{p}} K$. The $K$-algebra $A^{\prime}$ is generated in filtration degree 1 , and $L:=F_{1} A^{\prime}$ is a finite dimensional vector space over $K$ (as it is spanned by $1, a_{1}, \ldots, a_{n}$ over $A[0] \otimes_{A[0]^{p}} K$, and 
$A[0]$ is module-finite over $A[0]^{p}$ as $A[0]$ is a finitely generated algebra). Also, $L$ is closed under commutator. Thus, $L$ is a finite dimensional Lie algebra over $K$, and $A^{\prime}$ is a quotient of the enveloping algebra $U(L)$. But the enveloping algebra of a finite dimensional Lie algebra in characteristic $p$ is module-finite over its center (i.e., there is a $p$-center; see [Jacobson 1952; 1962, Chapter 6, Lemma 5]). This implies that $A^{\prime}$ is module-finite over its center, as desired.

(ii) Since a $W$-algebra is a quantum Hamiltonian reduction of the enveloping algebra $U(\mathfrak{g})$ of a semisimple Lie algebra $\mathfrak{g}$ [Losev 2010], the statement follows from (i).

(iii) This also follows from (i) and the definition of the quantum Hamiltonian reduction.

(iv) This holds by [Etingof 2006, Theorem 9.1.1 (in the appendix)].

(v) This follows easily from the previous cases.

\section{Finite dimensional Hopf actions on filtered quantizations}

3A. Hopf-Galois actions. Theorem 2.4 does not hold for nonsemisimple Hopf actions, as there are many inner faithful actions of nonsemisimple finite dimensional Hopf algebras on commutative domains; see [Etingof and Walton 2015]. However, Theorem 2.4 is valid in the Hopf-Galois case.

Theorem 3.1. Let $B$ be a filtered quantization of a commutative finitely generated domain with PI reductions, and let $H$ be a finite dimensional Hopf algebra over $k$ which acts on $B$. Assume that this action gives rise to an $H^{*}$-Hopf-Galois extension $B^{H} \subset B$. Then $H$ is a group algebra.

Proof. The result follows from the arguments in the proofs of Theorem 2.4 and [Cuadra et al. 2015, Theorem 4.2]. Namely, recall Notation 1.3. We show, similarly to the proof of Theorem 2.4, that $Z$ is $H_{p}$-stable, and then proceed as in the proof of [Cuadra et al. 2015, Theorem 4.2]. Specifically, by [Etingof 2016, Corollary 3.2(ii)], $D_{p}$ has degree $p^{n}$ over its center $Z=Z\left(D_{p}\right)$ for some $n$, so by [Cuadra et al. 2015, Proposition 3.3(i)], $Z$ is $H_{p}$-invariant. Now, since the action of $H$ on $B$ gives rise to a Hopf-Galois extension, so does the action of $H_{p}$ on $Z$, i.e., the algebra map $Z \otimes_{Z^{H_{p}}} Z \rightarrow Z \otimes H_{p}^{*}$ is an isomorphism. Thus, $H_{p}^{*}$ is commutative and $H_{p}$ is cocommutative, so $H$ is cocommutative [Cuadra et al. 2015, Lemma 2.3(ii)], i.e., a group algebra by the Cartier-Gabriel-Kostant theorem [Montgomery 1993, Corollary 5.6.4(3) and Theorem 5.6.5].

3B. Preparatory results on nondegenerate quantizations. Another generalization of Theorem 2.4 concerns nondegenerate quantizations, defined in Definition 1.4. To obtain it, we first need to generalize [Cuadra et al. 2016, Theorem 3.2]. Let $\mathcal{H}$ be a finite dimensional Hopf algebra over an algebraically closed field $F$ of 
characteristic $p>0$, and let $\mathcal{Z}$ be a finitely generated field extension of $F$. Let $\mathcal{Z}(m)$, for $m \geq 1$, be a collection of subfields of $\mathcal{Z}$ such that $\mathcal{Z}(m) \supset \mathcal{Z}(m+1)$ for all $m \geq 1$.

Theorem 3.2. Suppose that $\bigcap_{m \geq 1} \mathcal{Z}(m)=F$, and that $[\mathcal{Z}: \mathcal{Z}(m)]$ is a power of $p$ for all $m \geq 1$. Assume that $\mathcal{H}$ acts $F$-linearly and inner faithfully on $\mathcal{Z}$. If $p>\operatorname{dim} \mathcal{H}$ and $\mathcal{H}$ preserves $\mathcal{Z}(m)$ for all $m$, then $\mathcal{H}$ is a group algebra.

Proof. The proof is the same as that of [Cuadra et al. 2016, Theorem 3.2]. Indeed, the only properties of the fields $Z^{p^{m}}$ used in that proof are that their intersection is $F$ and that the degree of $Z$ over $Z^{p^{m}}$ is a power of $p$.

We will also need the lemma below from commutative algebra. We first introduce the following notation. Let $W_{N}=W_{N}(F):=W(F) /\left(p^{N}\right)$ be the $N$-th truncated Witt ring of $F\left(W_{N}\right.$ is an algebra over $\mathbb{Z} / p^{N} \mathbb{Z}$; cf. [Cuadra et al. 2016, Subsection 2.1]). Let $Y$ be an irreducible smooth affine algebraic variety over $F$ with structure algebra $A:=O(Y)$, and $\widetilde{Y}$ be a flat deformation of $Y$ over $W_{N}$. Let $1 \leq m \leq N$, and let $A_{m}:=O(\widetilde{Y}) /\left(p^{m}\right)\left(\right.$ a free $\mathbb{Z} / p^{m} \mathbb{Z}$-module); thus $A_{1}=A$ and $A_{m-1}=A_{m} /\left(p^{m-1}\right)$ for $m \geq 2$. Let

$$
d_{m}: A_{m} \rightarrow \Omega_{A_{m} / W_{m}}
$$

be the differential.

\section{Lemma 3.3. For $1 \leq m \leq N$, the image of $\operatorname{ker}\left(d_{m}\right)$ in $A$ is $A^{p^{m}}$.}

Proof. It is clear that the image of $\operatorname{ker}\left(d_{m}\right)$ contains $A^{p^{m}}$, so it remains to establish the opposite inclusion. We do so by induction in $m$.

The base of induction is the equality $\operatorname{ker}\left(\left.d\right|_{A}\right)=A^{p}$, which is the Cartier isomorphism in degree zero [Katz 1970, Section 7]. Alternatively, here is a direct proof. Since $A$ is integrally closed in its quotient field $L:=\operatorname{Frac}(A)$, we may replace $A$ with $L$. Note that $L$ can be represented as a finite separable extension of $F\left(y_{1}, \ldots, y_{n}\right)$, where $n=\operatorname{dim} Y$. Given $f \in L$ such that $d f=0$, consider the minimal polynomial $P(t)=t^{r}+a_{r-1} t^{r-1}+\cdots+a_{0}$ of $f$ over $E:=F\left(y_{1}, \ldots, y_{n}\right)$. Applying the differential to the equation $P(f)=0$, we get $\sum_{j=0}^{r-1} f^{j} d a_{j}=0$. Since $P$ is the minimal polynomial, this implies that $d a_{j}=0$ for all $j$. Thus $a_{j} \in E^{p}$ (as the statement in question is easy for purely transcendental fields). Thus, $E^{p}(f)$ is a finite separable extension of $E^{p}$ (as $P$ is a separable polynomial). But $E^{p}(f)$ is a purely inseparable extension of $E^{p}\left(f^{p}\right)$. Hence, $E^{p}(f)=E^{p}\left(f^{p}\right)$, that is, $f \in E^{p}\left(f^{p}\right) \subset L^{p}$, as desired.

To perform the induction step, suppose $f \in \operatorname{ker}\left(d_{m}\right)$. Our job is to show that the image $\bar{f}$ of $f$ in $A$ is contained in $A^{p^{m}}$. By the induction assumption we know that $\bar{f}=b^{p^{m-1}}$ for some $b \in A$, so it remains to show that $b=c^{p}$ for some $c \in A$.

For this, we expand $f$ in a power series in some local coordinate system $y_{1}, \ldots, y_{n}$ on $\tilde{Y}$. It is easy to see by looking at monomials that if $g \in W_{m} \llbracket y_{1}, \ldots, y_{n} \rrbracket$ 
and $d g=0$, then the reduction $\bar{g}$ of $g$ modulo $p$ lies in $F \llbracket y_{1}^{p^{m}}, \ldots, y_{n}^{p^{m}} \rrbracket$. In particular, $F \llbracket y_{1}^{p^{m}}, \ldots, y_{n}^{p^{m}} \rrbracket$ contains the power series expansion of $\bar{f}$ in $y_{i}$. This means that the power series expansion of $b$ is in $F \llbracket y_{1}^{p}, \ldots, y_{n}^{p} \rrbracket$. Thus, $d b=0$. By the base of induction we conclude that $b=c^{p}$ for some $c \in A$, which completes the induction step.

Moreover, we will need the result below.

Lemma 3.4. Let $B$ be an algebra with PI reductions, and let $D_{p}$ denote the full localization (i.e., the ring of fractions) of the reduction $B_{p}$ of $B$, for $p \gg 0$. Then the center $Z$ of $D_{p}$ is a finitely generated field extension of $\overline{\mathbb{F}}_{p}$.

Proof. Let $v_{1}, \ldots, v_{N}$ be a basis of $D_{p}$ over $Z$, and let $b_{1}, \ldots, b_{n}$ be generators of $B_{p}$. Then $b_{s} v_{i}=\sum_{j=1}^{N} \beta_{s i}^{j} v_{j}$ for $\beta_{s i}^{j} \in Z$. Let $K$ denote the field $\overline{\mathbb{F}}_{p}\left(\beta_{s i}^{j}\right)$.

Now take $z \in Z$. Then $z \in D_{p}$, so $z=c^{-1} b$, and hence $c z=b$ for some $b, c \in B_{p}$ with $c \neq 0$. Since $b, c \in B_{p}$, they are noncommutative polynomials in $b_{1}, \ldots, b_{n}$ over $\overline{\mathbb{F}}_{p}$. So, $b v_{i}=\sum \beta_{i}^{j} v_{j}, c v_{i}=\sum \gamma_{i}^{j} v_{j}$, with $\beta_{i}^{j}, \gamma_{i}^{j} \in K$. But $\gamma_{i}^{j} z=\beta_{i}^{j}$ and $\gamma_{i}^{j}$ are not all zero. So, $z \in K$ and hence $Z=K$. Thus, $Z$ is a finitely generated extension of $\overline{\mathbb{F}}_{p}$.

3C. Hopf actions on nondegenerate quantizations. Now let $B$ be a filtered quantization with PI reductions.

Theorem 3.5. If B is a nondegenerate algebra with PI reductions, then any finite dimensional Hopf action on B factors through a group action (i.e., the condition that $H$ is semisimple in Theorem 2.4 can be dropped).

Proof. The proof is obtained by combining the proofs of Theorem 2.4 and [Cuadra et al. 2016, Theorem 1.1] with the following modifications.

1. In [Cuadra et al. 2016, Lemma 2.5] and below, $x_{i}, y_{i}$ should be replaced by any finite set of generators $L_{1}, \ldots, L_{r}$ of $B$, and the number $2 n$ in the proof of [Cuadra et al. 2016, Lemma 4.3] should be replaced by $r$ (cf. [Cuadra et al. 2016, proof of Theorem 1.2]).

2. The discussion in [Cuadra et al. 2016, Subsection 2.4, Lemma 4.7, Proposition 4.8] (needed to justify the assumptions of [Cuadra et al. 2016, Theorem 3.2]) becomes unnecessary. Instead, note that if $a \in D_{p^{m}}$ is central modulo $p^{m-1}$ for some $m \geq 2$, then $a^{p}$ is central. Hence $Z(m) \supset Z(m-1)^{p}$, implying that $Z(m) \supset Z^{p^{m-1}}$ and therefore $[Z: Z(m)]$ is finite (by Lemma 3.4) and is a power of $p$. Now the proof proceeds by invoking Theorem 3.2, whose assumptions are satisfied by the nondegeneracy property of $B$ and using a straightforward generalization of [Cuadra et al. 2016, Lemma 4.6]. 
3. The rest of the proof of [Cuadra et al. 2016, Theorem 1.1] is modified as in the proof of Theorem 2.4. Namely, we use the PI reduction condition and [Etingof 2016, Corollary 3.2], which says that the PI degree of $B_{p}$ is a power of $p$.

The next theorem shows that the nondegeneracy assumption is satisfied, in particular, when $\operatorname{gr}(B)=O(X)$, where $X$ is generically symplectic.

Theorem 3.6. Let $B$ be a quantization with PI reductions. Assume $\operatorname{gr}(B)=O(X)$, where $X$ is a generically symplectic Poisson variety. Then any action of a finite dimensional Hopf algebra $H$ on $B$ factors through a group action.

Proof. By Theorem 3.5, it suffices to show that $B$ is a nondegenerate quantization, i.e., that $\bigcap_{m \geq 1} Z(m)=\overline{\mathbb{F}}_{p}$ for $p \gg 0$.

Recall Notation 1.3. Let $C$ be the center of $B_{p}$; thus, by Posner's theorem [McConnell and Robson 2001, Theorem 13.6.5], the field $\operatorname{Frac}(C)$ of fractions of $C$ is $Z$. Let $C_{m}$ be the center of $B_{p^{m}}$, and $C(m)$ be its image in $B_{p}$.

Let $a \in B_{p^{m}}$ be central modulo $p$ (i.e., the image $\bar{a}$ of $a$ in $B_{p}$ lies in $C$ ). Then $a^{p}$ is central modulo $p^{2}, a^{p^{2}}$ is central modulo $p^{3}$, and so on. Hence, $C^{p^{m-1}} \subset C(m)$. Let $C_{m}^{\prime}$ be the preimage of $C^{p^{m-1}}$ in $C_{m}$. Then the image of $C_{m}^{\prime}$ in $B_{p}$ is $C^{p^{m-1}}$.

We claim that

$$
Z(m)=\operatorname{Frac}(C(m)) .
$$

Indeed, it is clear that $\operatorname{Frac}(C(m)) \subseteq Z(m)$. On the other hand, observe that any element $a \in D_{p^{m}}$ can be written as $a=c^{-1} b$, where $c \in C_{m}^{\prime}$ is nonzero modulo $p$, and $b \in B_{p^{m}}$ (as this can be done modulo $p$, since $D_{p}=Z^{p^{m-1}} B_{p}$ ). Now given $z \in Z(m)$, let $\tilde{z}$ be its lift to $Z_{m}$. Writing $\tilde{z}=c^{-1} b$ as above, we see that $b:=c \tilde{z} \in C_{m}$. Let $\bar{b} \in C(m)$ and $\bar{c} \in C^{p^{m-1}} \subset C(m)$ be the reductions of $b$ and $c$ modulo $p$, respectively. We have $\bar{b}=\bar{c} z$, hence $z=\bar{c}^{-1} \bar{b} \in \operatorname{Frac}(C(m))$, as claimed.

Now let $B_{0 p^{m}}:=\operatorname{gr}\left(B_{p^{m}}\right)$. This is a Poisson algebra over the truncated Witt ring $W_{m, p}$. Let $C_{0 m}$ be the Poisson center of $B_{0 p^{m}}$, and $C_{0}(m)$ be the image of $C_{0 m}$ in $B_{0 p}$. Then $\operatorname{gr}\left(C_{m}\right) \subset C_{0 m}$ and hence

$$
\operatorname{gr}(C(m)) \subset C_{0}(m) .
$$

Let $Z_{0}:=\operatorname{Frac}\left(B_{0 p}\right)$. Since $X$ is generically symplectic, $C_{0 m}$ coincides with the set of all $f \in B_{0 p^{m}}$ such that $d f=0$. By Lemma 3.3 (taking $\widetilde{Y}$ to be the reduction modulo $p^{m}$ of a symplectic dense affine open subset $U \subset X$ ), this implies that

$$
\operatorname{Frac}\left(C_{0}(m)\right) \subset Z_{0}^{p^{m}} .
$$

Now suppose that $z \in \bigcap_{m \geq 1} Z(m)$ with $z \neq 0$. Then by (3), for each $m$, we have $z=f_{m} / g_{m}$ for $f_{m}, g_{m} \in C(m)$. Let $f_{m}^{0}, g_{m}^{0} \in \operatorname{gr}(C(m))$ be the leading terms of $f_{m}, g_{m}$. By (4), $f_{m}^{0}, g_{m}^{0} \in C_{0}(m)$. Then for any $m, n$ we have $f_{m}^{0} g_{n}^{0}=f_{n}^{0} g_{m}^{0}$ since $f_{m} g_{n}=f_{n} g_{m}$. So $z_{0}:=f_{m}^{0} / g_{m}^{0}$ is independent of $m$ and by (5) belongs to $Z_{0}^{p^{m}}$ for all $m \geq 0$. As $\bigcap_{m \geq 1} Z_{0}^{p^{m}}$ is a perfect field that is finitely generated over $\overline{\mathbb{F}}_{p}$, we get 
that $\bigcap_{m \geq 1} Z_{0}^{p^{m}}=\overline{\mathbb{F}}_{p}$. So, $z_{0} \in \overline{\mathbb{F}}_{p}$ is a nonzero constant, and $f_{m}^{0}=z_{0} g_{m}^{0}$ for all $m$; in particular,

$$
\operatorname{deg}\left(f_{m}\right)=\operatorname{deg}\left(g_{m}\right)
$$

Now $z-z_{0}=\left(f_{m}-z_{0} g_{m}\right) / g_{m}$, and the numerator has degree strictly less than deg $g_{m}$. This violates (6), so $z-z_{0}=0$, i.e., $z \in \overline{\mathbb{F}}_{p}$. This proves the theorem.

Corollary 3.7. Let $B$ be one of the following algebras:

(i) a quotient of a finite $W$-algebra by a central character;

(ii) a Hamiltonian reduction of a Weyl algebra by a reductive group action; in particular, the coordinate ring of a quantized quiver variety;

(iii) a spherical symplectic reflection algebra $H_{1, c}(G, V)$; or

(iv) the tensor product of any of the algebras in (i)-(iii).

Then any action of a finite dimensional Hopf algebra $H$ on $B$ factors through a group action.

Proof. It is explained in Section 2B that in examples (i)-(iv), we have $\operatorname{gr}(B)=O(X)$, where $X$ is generically symplectic. This implies the corollary.

Proposition 3.8. Theorems $2.4,3.1,3.5$, and 3.6 remain valid if $B$ is replaced by its quotient division algebra $\operatorname{Frac}(B)$.

Proof. The proofs are obtained by combining the proofs of Theorems 2.4, 3.1, 3.5, and 3.6 with the proof of [Cuadra et al. 2015, Proposition 4.4]. (The exact form of the generators of $B$ used in the proof of [Cuadra et al. 2015, Proposition 4.4] is irrelevant for the argument.)

\section{Semisimple Hopf actions on quantum polynomial algebras}

We now turn to finite dimensional Hopf actions on quantum polynomial algebras

$$
k_{q}\left[x_{1}, \ldots, x_{n}\right]:=k\left\langle x_{1}, \ldots, x_{n}\right\rangle /\left(x_{i} x_{j}-q_{i j} x_{j} x_{i}\right),
$$

where $\boldsymbol{q}=\left(q_{i j}\right), q_{i j} \in k^{\times}$with $q_{i i}=1$ and $q_{i j} q_{j i}=1$. We view $\boldsymbol{q}$ as a point of the algebraic torus $\left(k^{\times}\right)^{n(n-1) / 2}$ with coordinates $q_{i j}, i<j$. Let $\langle\boldsymbol{q}\rangle$ be the subgroup in $\left(k^{\times}\right)^{n(n-1) / 2}$ generated by $\boldsymbol{q}$, and let $G_{\boldsymbol{q}}$ be its Zariski closure. Let $G_{\boldsymbol{q}}^{0}$ be the connected component of the identity in $G_{q}$.

Theorem 4.1. Let $H$ be a semisimple Hopf algebra of dimension d. If the order of $G_{\boldsymbol{q}} / G_{\boldsymbol{q}}^{0}$ is coprime to $d !$, then any $H$-action on $B:=k_{\boldsymbol{q}}\left[x_{1}, \ldots, x_{n}\right]$ factors through a group action.

Proof. We may assume that $H$ acts on $B:=k_{q}\left[x_{1}, \ldots, x_{n}\right]$ inner faithfully. Let $R \subset k$ be a finitely generated subring containing $q_{i j}$, let $B_{R}:=R_{q}\left[x_{1}, \ldots, x_{n}\right]$ be 
the quantum polynomial algebra defined over $R$, and let $H_{R}$ be a Hopf $R$-order with an action on $B_{R}$ which becomes the action of $H$ on $B$ upon tensoring with $k$.

Similarly to the proof of Theorem 2.4, we need to control the PI degree of $B_{R}$ after reducing modulo $p$; we employ a version of the number-theoretic result of A. Perucca (as presented in the Appendix) to do so.

Given a number field $K$ and a ring homomorphism $\xi: R \rightarrow K$, let $R^{\prime}:=\xi(R)$, $H_{R^{\prime}}:=H_{R} \otimes_{R} R^{\prime}, B_{R^{\prime}}:=B_{R} \otimes_{R} R^{\prime}=R_{\xi(\boldsymbol{q})}^{\prime}\left[x_{1}, \ldots, x_{n}\right]$. Then $H_{R^{\prime}}$ acts on $B_{R^{\prime}}$ inner faithfully. For a generic choice of $\xi$, any multiplicative relation satisfied by $\xi\left(q_{i j}\right)$ is already satisfied by $q_{i j}$, so by Example A.3, we have $\left|G_{\boldsymbol{q}} / G_{\boldsymbol{q}}^{0}\right|=\left|G_{\xi(\boldsymbol{q})} / G_{\xi(\boldsymbol{q})}^{0}\right|$. By Corollary A.2, there exist infinitely many primes $p$ with prime ideals $\mathfrak{p} \subset R^{\prime}$ lying over them such that, for a generic homomorphism $\psi: R^{\prime} \rightarrow \overline{\mathbb{F}}_{p}$ annihilating $\mathfrak{p}$, the order $N:=N_{\mathfrak{p}}$ of $\psi \circ \xi(\boldsymbol{q})$ is finite and relatively prime to $d$ !. Let $H_{p}:=H_{R^{\prime}} \otimes_{R^{\prime}} \overline{\mathbb{F}}_{p}$ and $B_{p}:=B_{R^{\prime}} \otimes_{R^{\prime}} \overline{\mathbb{F}}_{p}$ be the corresponding reductions of $H$ and $B$ modulo $p$. For large enough $p$, the Hopf algebra $H_{p}$ is semisimple and cosemisimple by [Cuadra et al. 2015, Lemma 2.5], and $B_{p}$ is a PI domain with PI degree dividing $N^{n}$ (as $x_{i}^{N}$ are central elements in $B_{p}$ ). Moreover, $H_{p}$ acts on $B_{p}$ inner faithfully by a version of [Cuadra et al. 2015, Proposition 2.4] adapted to the algebra $B$ (with the same proof).

Let $D_{p}$ be the quotient division algebra of $B_{p}$. Then the PI degree of $D_{p}$ divides $N^{n}$, and is therefore coprime to $d$ !. Further, $H_{p}$ acts inner faithfully on $D_{p}$. Hence, [Cuadra et al. 2015, Proposition 3.3(ii)] implies that $H_{p}$ is cocommutative. Since this happens for infinitely many primes, we conclude that $H_{R^{\prime}}$ is cocommutative. Since this happens for generic maps $\xi$, this implies that $H_{R}$ is cocommutative. Thus $H$ is cocommutative, i.e., $H$ is a group algebra.

Corollary 4.2. The conclusion of Theorem 4.1 holds when $q_{i j}=q^{m_{i j}}$, where $m_{i j}=-m_{j i}$ are integers, and the order of $q \in k^{\times}$is infinite or is coprime to $d$ !.

Proof. This is a special case of Theorem 4.1.

Example 4.3. The assumption in Theorem 4.1 and Corollary 4.2 that the order of $G_{q} / G_{q}^{0}$ is coprime to $d$ ! cannot be removed. For instance, there exists an inner faithful action of the 8-dimensional noncommutative noncocommutative semisimple Hopf algebra on the quantum polynomial algebra $k_{-1}[x, y]$; see [Kirkman et al. 2009, Example 7.6]. In this case, $\left|G_{\boldsymbol{q}} / G_{\boldsymbol{q}}^{0}\right|=2$.

\section{Finite dimensional Hopf actions on quantum polynomial algebras}

Let us now extend the results of the previous section to not necessarily semisimple Hopf algebras, under some additional assumptions.

First of all, when the action of $H$ on $B$ is Hopf-Galois, we can remove in Theorem 4.1 the assumption that $H$ is semisimple, and also weaken the coprimeness assumption, replacing $d$ ! with $d$. 
Proposition 5.1. Suppose that a finite dimensional Hopf algebra $H$ acts on $B:=$ $k_{q}\left[x_{1}, \ldots, x_{n}\right]$, and the order of $G_{q} / G_{q}^{0}$ is coprime to $d$. If this action gives rise to an $H^{*}-$ Hopf-Galois extension $B^{H} \subset B$, then the action of $H$ on $B$ factors through a group algebra.

Proof. The proof is parallel to the proof of Theorem 3.1. The weaker coprimeness assumption suffices since by the Hopf-Galois condition, $\left[D_{p}: D_{p}^{H}\right]=d$ (not just $\leq d$ ). Here, $p \gg 0$ and $D_{p}$ is the full localization of $B$ reduced modulo $p$ via the method in the proof of Theorem 4.1.

Let us now give a generalization of Theorem 4.1 to the nonsemisimple case under a nondegeneracy assumption.

Theorem 5.2. Let $H$ be a finite dimensional Hopf algebra of dimension d acting on $B:=k_{\boldsymbol{q}}\left[x_{1}, \ldots, x_{n}\right]$. Assume that the order of $G_{\boldsymbol{q}} / G_{\boldsymbol{q}}^{0}$ is coprime to $d !$, and $\boldsymbol{q}$ is nondegenerate. Then the action of $H$ on $B$ factors through a group action.

Proof. The proof is obtained by combining the proofs of Theorems 3.5 and 4.1. Let us describe the necessary changes.

We argue as in the proof of Theorem 4.1. Fix a generic character $\xi: R \rightarrow K$ from $R$ to a number field $K$, and set $R^{\prime}=\xi(R)$. By Corollary A.2, there exist infinitely many primes $p$ with prime ideals $\mathfrak{p} \subset R^{\prime}$ lying over them such that, for a generic homomorphism $\psi: R^{\prime} \rightarrow \overline{\mathbb{F}}_{p}$ annihilating $\mathfrak{p}$, the order $N:=N_{\mathfrak{p}}$ of $\psi \circ \xi(\boldsymbol{q})$ is finite and coprime to $d$ !.

Consider the image $Z(m)$ of the center $Z_{m}$ of $D_{p^{m}}$ in $D_{p}$ (thus, $Z(1)=Z$ ). By a straightforward generalization of [Cuadra et al. 2016, Lemma 4.6], $Z(m)$ is preserved by the action of $H_{p}$. It is clear that $Z(m)$ is generated by the monomials $x_{1}^{m_{1}} \cdots x_{n}^{m_{n}}$ such that $\prod_{j} q_{i j}^{m_{j}}=1$ in the truncated ring of Witt vectors $W_{m, p}$ (see [Cuadra et al. 2016, Section 2.1]). Let $W_{m, p}^{\prime}$ be the kernel of the natural map of multiplicative groups $W_{m, p}^{\times} \rightarrow \overline{\mathbb{F}}_{p}^{\times}$. Then every element of $W_{m, p}^{\prime}$ has order a power of $p$. Hence, $[Z: Z(m)]$ is a power of $p$. Also it is clear from the nondegeneracy condition for $\boldsymbol{q}$ that $\bigcap_{m} Z(m)=\overline{\mathbb{F}}_{p}$. Thus, Theorem 3.2 applies, and yields that $H_{p}$ is cocommutative. Hence $H_{R^{\prime}}$ is cocommutative, implying that $H_{R}$ is cocommutative and ultimately that $H$ is cocommutative, i.e., a group algebra.

Remark 5.3. If $q_{i j}=q^{m_{i j}}$, where $q$ is not a root of unity, then $\boldsymbol{q}$ is nondegenerate if and only if $\operatorname{det}\left(m_{i j}\right) \neq 0$. Theorem 5.2 applies in this case. This gives a generalization of [Chan et al. 2014, Theorem 0.4] to nonhomogeneous Hopf actions for even $n$.

Proposition 5.4. Theorem 4.1, Corollary 4.2, and Theorem 5.2 remain valid if the quantum polynomial algebra $B$ is replaced by the quantum torus $k_{q}\left[x_{1}^{ \pm 1}, \ldots, x_{n}^{ \pm 1}\right]$ or the division algebra of quotients $\operatorname{Frac}(B)$. 
Proof. In the case of the quantum torus, the proof is analogous to the proof of Theorem 4.1. The case of the division algebra of quotients is obtained using the same argument as in the proof of [Cuadra et al. 2015, Proposition 4.4].

Example 5.5. The condition that $H$ is semisimple cannot be dropped in Theorem 4.1, and the condition that $\boldsymbol{q}$ is nondegenerate cannot be dropped in Theorem 5.2.

Namely, let $A=A_{0} \oplus A_{1}$ be a $\mathbb{Z} / 2 \mathbb{Z}$-graded domain with a nonzero central element $z \in A_{1}$, and take $H$ to be the 4-dimensional Sweedler Hopf algebra generated by a group-like element $g$ and a $(g, 1)$-skew-primitive element $u$ with $g^{2}=1, u^{2}=0$ and $g u+u g=0$.

(1) Then there is an action of $H$ on $A$ (not preserving the grading of $A$ ) given by $g \cdot a=(-1)^{\operatorname{deg} a} a$, and $u \cdot a=0$ if $a \in A_{0}$ and $u \cdot a=z a$ if $a \in A_{1}$. It is easy to check that this action is well-defined, and it is inner faithful since $u$ acts by a nonzero operator.

(2) In particular, we have an inner faithful action of $H$ on the quantum polynomial algebra $k_{q}[x, y]$, for $q$ a root of unity of any odd order $2 m-1, m>0$; namely, we can take $z=x^{2 m-1}$.

(3) This gives an inner faithful action of $H$ on the quantum torus $k_{q}\left[x_{1}^{ \pm 1}, \ldots, x_{n}^{ \pm 1}\right]$ if $n$ is odd: we can take the central element

$$
z=x_{1} x_{2}^{-1} x_{3} \cdots x_{n-1}^{-1} x_{n}
$$

For even $n$, such an action is impossible if $q$ is not a root of unity by Theorem 5.2. Indeed, the matrix $m_{i j}:=\operatorname{sign}(j-i)$ is nondegenerate if and only if $n$ is even (see Remark 5.3).

(4) Finally, this gives an inner faithful Sweedler Hopf algebra action on the Weyl algebra $\boldsymbol{A}_{n}(F)$ when $\operatorname{char}(F)=p \geq 3$; the $\mathbb{Z} / 2 \mathbb{Z}$ grading is defined by giving the generators degree 1 , and we can take, for instance, $z=x_{1}^{p}$. (Note that by [Cuadra et al. 2016, Theorem 1.1], this is impossible in characteristic zero; indeed, the center of $\boldsymbol{A}_{n}(k)$ is $k$.)

\section{Semisimple Hopf actions on twisted homogeneous coordinate rings and 3-dimensional Sklyanin algebras}

Now let us consider semisimple Hopf actions on twisted homogeneous coordinate rings of abelian varieties. We keep the notation of Section 1D.

Let $H$ be a Hopf algebra over $k$ of dimension $d$.

Theorem 6.1. We have the following statements.

(i) If $H$ is semisimple, and if the order of $G_{\sigma} / G_{\sigma}^{0}$ is coprime to d!, then any $H$-action on $B:=B(X, \sigma, \mathcal{L})$ factors through a group action. 
(ii) Moreover, part (i) holds for the H-action on the division algebra of quotients $\operatorname{Frac}(B)$ of $B$.

(iii) Part (i) also holds for not necessarily semisimple $H$ if the order of $G_{\sigma} / G_{\sigma}^{0}$ is coprime to $d$ and the $H$-action gives rise to a Hopf-Galois extension.

Proof. The proofs of the statements (i)-(iii) are parallel to the proofs of Theorem 4.1, Proposition 5.4, and Proposition 5.1, respectively, where we use that the PI degree of $B$ equals the order of $\sigma$. The only difference is that Corollary A.2 is applied to the abelian variety $X$ with subgroup $\left\{s^{i}\right\}_{i \in \mathbb{Z}}$ rather than the torus $\left(k^{\times}\right)^{n(n-1) / 2}$ with subgroup $\langle\boldsymbol{q}\rangle$.

In particular, if $X=: E$ is an elliptic curve, Theorem 6.1 holds if the order of $\sigma$ is coprime to $d$ ! or infinite. Moreover, if $\sigma$ has infinite order, the assumption that $H$ is semisimple can be dropped.

Theorem 6.2. Let $E$ be an elliptic curve, and take $\sigma \in A u t(E)$ given by translation by a point of infinite order. Then any finite dimensional Hopf action on $B(E, \sigma, \mathcal{L})$ factors through a group action.

Proof. The proof repeats the proofs of Theorems 5.2 and 3.5 without significant changes.

Finally, let us consider semisimple Hopf actions on 3-dimensional Sklyanin algebras [Artin et al. 1990; Odesskiı and Fer̆gin 1989]. Let $F$ be an algebraically closed field of characteristic not equal to 2 or 3 .

Definition 6.3. Let $a, b, c \in F^{\times}$be such that

$$
(3 a b c)^{3} \neq\left(a^{3}+b^{3}+c^{3}\right)^{3} .
$$

The 3-dimensional Sklyanin algebra, denoted by $S(a, b, c)$ is generated over $F$ by $x, y, z$ with defining relations

$$
a y z+b z y+c x^{2}=a z x+b x z+c y^{2}=a x y+b y x+c z^{2}=0 .
$$

It is known that $S(a, b, c)$ is Koszul with Hilbert series $(1-t)^{-3}$ (see [Artin et al. 1990, Theorems 6.6(ii) and 6.8(i)] and a result of J. Zhang [Smith 1996, Theorem 5.11]), so that $S(a, b, c)$ is a flat deformation of the algebra of polynomials in three variables (see, e.g., [Tate and Van den Bergh 1996, Theorem 1.1]). Moreover, the center of $S(a, b, c)$ contains an element $T$ of degree 3 , and $S(a, b, c) /(T)=$ $B(E, \sigma, \mathcal{L})$, where $E$ is the elliptic curve in $\mathbb{P}^{2}$ given by the equation

$$
\left(a^{3}+b^{3}+c^{3}\right) x y z=a b c\left(x^{3}+y^{3}+z^{3}\right),
$$

$\sigma$ is given by translation by the point $(a: b: c) \in E$, and $\mathcal{L}$ is a line bundle of degree 3 on $E$. 
Theorem 6.4. Let $S(a, b, c)$ be a 3-dimensional Sklyanin algebra over $k$ and let $H$ be a semisimple Hopf algebra over $k$ of dimension d. If the order of $\sigma \in A u t(E)$ is coprime to $d !$ or infinite, then any $H$-action on $S(a, b, c)$ factors through a group action.

Proof. It is known from the theory of Sklyanin algebras that if $\sigma$ has order $N$, then $S(a, b, c)$ is PI with PI degree $N$ (see [Artin et al. 1994, part 5 of theorem on page 7]). Therefore, Theorem 6.4 is proved similarly to Theorem 4.1, using Corollary A.2 for elliptic curves, as in Theorem 5.2.

Remark 6.5. The semisimplicity condition on $H$ in Theorem 6.1 and the infinite order condition in Theorem 6.2 cannot be dropped, as there exists a Sweedler Hopf algebra action on $B:=B(X, \sigma, \mathcal{L})$ if $\sigma$ has odd order $N$. Namely, we take a sufficiently large odd number $m$ such that the line bundle $\mathcal{L}^{\otimes m}$ is very ample (it exists since $\mathcal{L}$ is ample). Now $B[m N] \neq 0$ and there exists an eigenvector $f$ of $\sigma$ in $B[m N]$. We then take $z=f^{N}$, a nonzero central element of odd degree $m N^{2}$, so that a desired action is given by Example 5.5.

Also, the semisimplicity assumption in Theorem 6.4 cannot be dropped, as there exists a Sweedler Hopf algebra action on $S(a, b, c)$ for any $a, b, c$, given by Example 5.5 where we use the central element $T$ in place of the element $z$.

\section{Appendix}

The goal of this Appendix is to provide number-theoretic results needed in Section 4. We start by quoting a result from [Perucca 2009] (in which we take $F$ to be the number field $K$ itself).

Theorem A.1 [Perucca 2009, Theorem 7]. Let $G$ be the product of an abelian variety and a torus defined over a number field $K$. Let $g \in G(K)$ be a $K$-rational point on $G$ such that the Zariski closure $G_{g}$ of the subgroup $\langle g\rangle \subset G(K)$ generated by $g$ is connected. Fix a positive integer $r$. Then there exists a positive Dirichlet density of primes $\mathfrak{p}$ of $K$ such that the order of $g$ modulo $\mathfrak{p}$ is coprime to $r$.

Corollary A.2. Let $K, G$ be as in Theorem A.1, let $g \in G(K)$, and let $\ell:=\left|G_{g} / G_{g}^{0}\right|$, where $G_{g}^{0}$ is the connected component of the identity in $G_{g}$ (i.e., $G_{g} / G_{g}^{0}=\mathbb{Z} / \ell \mathbb{Z}$ ). Fix a positive integer $r$ coprime to $\ell$. Then there exists a positive Dirichlet density of primes $\mathfrak{p}$ of $K$ such that the order of $g$ modulo $\mathfrak{p}$ is coprime to $r$.

The corollary above is used in the proof of Theorem 4.1, where $d$ ! is $r$ and $N_{\mathfrak{p}}$ is the order of $g$ modulo $\mathfrak{p}$.

Proof. The order of $g$ in $G_{g} / G_{g}^{0}$ is $\ell$, so $G_{g^{\ell}}=G_{g}^{0}$. Now the statement follows by applying Theorem A.1 to $g^{\ell}$.

Example A.3. Let $G$ be a split $m$-dimensional torus, and consider an element $g:=\left(q_{1}, \ldots, q_{m}\right) \in G$. We have the following statements. 
(1) The group $G_{g}$ is connected if and only if the group $\Gamma$ generated by $q_{1}, \ldots, q_{m}$ in $K^{\times}$is free, i.e., does not contain nontrivial roots of unity. Indeed both conditions are equivalent to the condition that any character $\chi$ of $G$ which maps $g$ to an $\ell$-th root of unity satisfies $\chi(g)=1$.

(2) More generally, $\left|G_{g} / G_{g}^{0}\right|=\ell$ if and only if the group of roots of unity generated by $\chi(g)$, where $\chi$ runs through characters of $G$ such that $\chi(g)$ is a root of unity, is $\mu_{\ell}$. In other words, $\ell$ is the order of the torsion subgroup in $\mathbb{Z}^{m} / g^{\perp}$, where $g^{\perp}$ is the subgroup of characters $\chi$ such that $\chi(g)=1$. In particular, $\ell$ depends only on the multiplicative relations satisfied by $q_{i j}$.

(3) If $\operatorname{dim} G=1$ (i.e., $G=\mathbb{G}_{m}$ or an elliptic curve), then $G_{g}$ is connected if and only if $g$ has infinite order or $g=1$. More generally, $\left|G_{g} / G_{g}^{0}\right|=\ell>1$ if and only if $g$ has order $\ell$.

\section{Acknowledgments}

We thank Bjorn Poonen for many useful discussions and for the number-theoretic reference [Perucca 2009], which is crucial for our arguments. We are also grateful to R. Bezrukavnikov, I. Losev, and H. Nakajima for useful discussions and explanations. We thank the referee for many useful comments that improved greatly the quality of this manuscript. The authors were supported by the National Science Foundation: NSF-grants DMS-1502244 and DMS-1550306.

\section{References}

[Artin and Van den Bergh 1990] M. Artin and M. Van den Bergh, "Twisted homogeneous coordinate rings", J. Algebra 133:2 (1990), 249-271. MR 1067406 Zbl 0717.14001

[Artin et al. 1990] M. Artin, J. Tate, and M. Van den Bergh, "Some algebras associated to automorphisms of elliptic curves", pp. 33-85 in The Grothendieck Festschrift, Vol. I, edited by P. Cartier et al., Progress in Mathematics 86, Birkhäuser, Boston, 1990. MR 1086882 Zbl 0744.14024

[Artin et al. 1994] M. Artin, W. Schelter, and J. Tate, "The centers of 3-dimensional Sklyanin algebras", pp. 1-10 in Barsotti Symposium in Algebraic Geometry (Abano Terme, 1991), edited by V. Cristante and W. Messing, Perspectives in Mathematics 15, Academic Press, San Diego, 1994. MR 1307390 Zbl 0823.17019

[Běllinson and Bernstein 1993] A. Bey̌linson and J. Bernstein, “A proof of Jantzen conjectures”, pp. 150 in I. M. Gel'fand Seminar, edited by S. Gel'fand and S. Gindikin, Advances in Soviet Mathematics 16, American Mathematical Society, Providence, RI, 1993. MR 1237825 Zbl 0790.22007

[Braden et al. 2012] T. Braden, N. Proudfoot, and B. Webster, "Quantizations of conical symplectic resolutions, I: local and global structure”, preprint, 2012. arXiv 1208.3863

[Chan et al. 2014] K. Chan, C. Walton, and J. Zhang, "Hopf actions and Nakayama automorphisms", J. Algebra 409 (2014), 26-53. MR 3198834 Zbl 1315.16027

[Chan et al. 2016] K. Chan, E. Kirkman, C. Walton, and J. J. Zhang, "Quantum binary polyhedral groups and their actions on quantum planes", J. Reine Angew. Math. 719 (2016), 211-252. MR 3552496 Zbl 06636678 
[Crawley-Boevey 2001] W. Crawley-Boevey, "Geometry of the moment map for representations of quivers”, Compositio Math. 126:3 (2001), 257-293. MR 1834739 Zbl 1037.16007

[Cuadra et al. 2015] J. Cuadra, P. Etingof, and C. Walton, "Semisimple Hopf actions on Weyl algebras", Adv. Math. 282 (2015), 47-55. MR 3374522 Zbl 06473182

[Cuadra et al. 2016] J. Cuadra, P. Etingof, and C. Walton, "Finite dimensional Hopf actions on Weyl algebras", Adv. Math. 302 (2016), 25-39. MR 3545923 Zbl 06631651

[Eisenbud 1995] D. Eisenbud, Commutative algebra, with a view toward algebraic geometry, Graduate Texts in Mathematics 150, Springer, 1995. MR 1322960 Zbl 0819.13001

[Etingof 2006] P. Etingof, “The $p$-center of symplectic reflection algebras”, (2006). Appendix to R. Bezrukavnikov, M. Finkelberg, and V. Ginzburg, "Cherednik algebras and Hilbert schemes in characteristic p", Represent. Theory 10 (2006), 254-298. MR 2219114 Zbl 1130.14005

[Etingof 2007] P. Etingof, Calogero-Moser systems and representation theory, European Mathematical Society, Zürich, 2007. MR 2296754 Zbl 1331.53002

[Etingof 2016] P. Etingof, "A PI degree theorem for quantum deformations”, J. Algebra 466 (2016), 308-313. MR 3541688 Zbl 06623498

[Etingof and Ginzburg 2010] P. Etingof and V. Ginzburg, "Noncommutative del Pezzo surfaces and Calabi-Yau algebras", J. Eur. Math. Soc. (JEMS) 12:6 (2010), 1371-1416. MR 2734346 Zbl 1204.14004

[Etingof and Walton 2014] P. Etingof and C. Walton, "Semisimple Hopf actions on commutative domains", Adv. Math. 251 (2014), 47-61. MR 3130334 Zbl 1297.16029

[Etingof and Walton 2015] P. Etingof and C. Walton, "Pointed Hopf actions on fields, I", Transform. Groups 20:4 (2015), 985-1013. MR 3416436 Zbl 1338.16035

[Etingof and Walton 2016] P. Etingof and C. Walton, "Finite dimensional Hopf actions on deformation quantizations", Proc. Amer. Math. Soc. (online publication October 2016).

[Grothendieck 1966] A. Grothendieck, "Éléments de géométrie algébrique, IV: Étude locale des schémas et des morphismes de schémas, III", Inst. Hautes Études Sci. Publ. Math. 28 (1966), 5-255. MR 0217086 Zbl 0144.19904

[Jacobson 1952] N. Jacobson, "A note on Lie algebras of characteristic p", Amer. J. Math. 74 (1952), 357-359. MR 0047026 Zbl 0046.03402

[Jacobson 1962] N. Jacobson, Lie algebras, Interscience Tracts in Pure and Applied Mathematics 10, Interscience Publishers, New York, 1962. MR 0143793 Zbl 0121.27504

[Katz 1970] N. M. Katz, "Nilpotent connections and the monodromy theorem: applications of a result of Turrittin”, Inst. Hautes Études Sci. Publ. Math. 39 (1970), 175-232. MR 0291177 Zbl 0221.14007

[Kirkman et al. 2009] E. Kirkman, J. Kuzmanovich, and J. J. Zhang, "Gorenstein subrings of invariants under Hopf algebra actions", J. Algebra 322:10 (2009), 3640-3669. MR 2568355 Zbl 1225.16015

[Lomp and Pansera 2015] C. Lomp and D. Pansera, "A note on a paper by Cuadra, Etingof and Walton”, preprint, 2015. To appear in Comm. Alg. arXiv 1506.07766

[Losev 2010] I. Losev, "Finite W-algebras", pp. 1281-1307 in Proceedings of the International Congress of Mathematicians (Hyderabad, 2010), vol. 3, edited by R. Bhatia et al., Hindustan Book Agency, New Delhi, 2010. MR 2827841 Zbl 1246.17015

[Losev and Etingof 2015] I. Losev and P. Etingof, "Bernstein inequality and holonomic modules", preprint, 2015. arXiv 1501.01260

[McConnell and Robson 2001] J. C. McConnell and J. C. Robson, Noncommutative Noetherian rings, revised ed., Graduate Studies in Mathematics 30, American Mathematical Society, Providence, RI, 2001. MR 1811901 Zbl 0980.16019 
[Montgomery 1993] S. Montgomery, Hopf algebras and their actions on rings, CBMS Regional Conference Series in Mathematics 82, American Mathematical Society, Providence, RI, 1993. MR 1243637 Zbl 0793.16029

[Nakajima 2015] H. Nakajima, "Towards a mathematical definition of Coulomb branches of 3dimensional $\mathcal{N}=4$ gauge theories, I”, preprint, 2015. arXiv 1503.03676

[Odesskiǔ 2002] A. V. Odesskiŭ, "Elliptic algebras", Uspekhi Mat. Nauk 57:6 (2002), 87-122. In Russian; translated in Russian Math. Surveys 57:6 (2002), 1127-1162. MR 1991863

[Odesskiı̌ and Feı̆gin 1989] A. V. Odesskiǔ and B. L. Feı̆gin, "Sklyanin's elliptic algebras", Funktsional. Anal. i Prilozhen. 23:3 (1989), 45-54. In Russian; translated in Funct. Anal. Appl. 23:3 (1989), 207-214. MR 1026987

[Ore 1931] O. Ore, "Linear equations in non-commutative fields", Ann. of Math. (2) 32:3 (1931), 463-477. MR 1503010 Zbl 0001.26601

[Perucca 2009] A. Perucca, "Prescribing valuations of the order of a point in the reductions of abelian varieties and tori”, J. Number Theory 129:2 (2009), 469-476. MR 2473894 Zbl 1166.14028

[Posner 1960] E. C. Posner, "Prime rings satisfying a polynomial identity", Proc. Amer. Math. Soc. 11 (1960), 180-183. MR 0111765 Zbl 0215.38101

[Sklyanin 1982] E. K. Sklyanin, "Some algebraic structures connected with the Yang-Baxter equation", Funktsional. Anal. i Prilozhen. 16:4 (1982), 27-34, 96. In Russian; translated in Funct. Anal. Appl. 16:4 (1983), 263-270. MR 684124 Zbl 0513.58028

[Skryabin and Van Oystaeyen 2006] S. Skryabin and F. Van Oystaeyen, "The Goldie Theorem for $H$-semiprime algebras”, J. Algebra 305:1 (2006), 292-320. MR 2264132 Zbl 1109.16033

[Smith 1996] S. P. Smith, "Some finite-dimensional algebras related to elliptic curves", pp. 315-348 in Representation theory of algebras and related topics (Mexico City, 1994), edited by R. Bautista et al., CMS Conf. Proc. 19, American Mathematical Society, Providence, RI, 1996. MR 1388568 Zbl 0856.16009

[Smith and Stafford 1992] S. P. Smith and J. T. Stafford, "Regularity of the four-dimensional Sklyanin algebra”, Compositio Math. 83:3 (1992), 259-289. MR 1175941 Zbl 0758.16001

[Stephenson 1997] D. R. Stephenson, "Algebras associated to elliptic curves", Trans. Amer. Math. Soc. 349:6 (1997), 2317-2340. MR 1390046 Zbl 0868.16028

[Tate and Van den Bergh 1996] J. Tate and M. Van den Bergh, "Homological properties of Sklyanin algebras", Invent. Math. 124:1 (1996), 619-647. MR 1369430 Zbl 0876.17010

Communicated by Susan Montgomery

Received 2016-05-19 Revised 2016-08-01 Accepted 2016-10-22

etingof@math.mit.edu

Department of Mathematics, Massachusetts Institute of Technology, 77 Massachusetts Avenue, Cambridge, MA 02139-4307, United States

notlaw@temple.edu

Department of Mathematics, Temple University, 1805

N. Broad Street, Philadelphia, PA 19122-6094, United States 


\section{Algebra \& Number Theory}

msp.org/ant

\section{EDITORS}

MANAGING EDITOR

Bjorn Poonen

Massachusetts Institute of Technology

Cambridge, USA

\author{
EDITORIAL BOARD CHAIR \\ David Eisenbud \\ University of California \\ Berkeley, USA
}

BOARD OF EDITORS

$\begin{aligned} \text { Dave Benson } & \text { University of Aberdeen, Scotland } & \text { Susan Montgomery } & \text { University of Southern California, USA } \\ \text { Richard E. Borcherds } & \text { University of California, Berkeley, USA } & \text { Shigefumi Mori } & \text { RIMS, Kyoto University, Japan } \\ \text { John H. Coates } & \text { University of Cambridge, UK } & \text { Raman Parimala } & \text { Emory University, USA } \\ \text { J-L. Colliot-Thélène } & \text { CNRS, Université Paris-Sud, France } & \text { Jonathan Pila } & \text { University of Oxford, UK } \\ \text { Brian D. Conrad } & \text { Stanford University, USA } & \text { Anand Pillay } & \text { University of Notre Dame, USA } \\ \text { Hélène Esnault } & \text { Freie Universität Berlin, Germany } & \text { Victor Reiner } & \text { University of Minnesota, USA } \\ \text { Hubert Flenner } & \text { Ruhr-Universität, Germany } & \text { Peter Sarnak } & \text { Princeton University, USA } \\ \text { Sergey Fomin } & \text { University of Michigan, USA } & \text { Joseph H. Silverman } & \text { Brown University, USA } \\ \text { Edward Frenkel } & \text { University of California, Berkeley, USA } & \text { Michael Singer } & \text { North Carolina State University, USA } \\ \text { Andrew Granville } & \text { Université de Montréal, Canada } & \text { Vasudevan Srinivas } & \text { Tata Inst. of Fund. Research, India } \\ \text { Joseph Gubeladze } & \text { San Francisco State University, USA } & \text { J. Toby Stafford } & \text { University of Michigan, USA } \\ \text { Roger Heath-Brown } & \text { Oxford University, UK } & \text { Ravi Vakil } & \text { Stanford University, USA } \\ \text { Craig Huneke } & \text { University of Virginia, USA } & \text { Michel van den Bergh } & \text { Hasselt University, Belgium } \\ \text { Kiran S. Kedlaya } & \text { Univ. of California, San Diego, USA } & \text { Marie-France Vignéras } & \text { Université Paris VII, France } \\ \text { János Kollár } & \text { Princeton University, USA } & \text { Kei-Ichi Watanabe } & \text { Nihon University, Japan } \\ \text { Yuri Manin } & \text { Northwestern University, USA } & \text { Efim Zelmanov } & \text { University of California, San Diego, USA } \\ \text { Philippe Michel } & \text { École Polytechnique Fédérale de Lausanne } & \text { Shou-Wu Zhang } & \text { Princeton University, USA }\end{aligned}$

PRODUCTION

production@msp.org

Silvio Levy, Scientific Editor

See inside back cover or msp.org/ant for submission instructions.

The subscription price for 2016 is US $\$ 290$ /year for the electronic version, and $\$ 485 /$ year (+\$55, if shipping outside the US) for print and electronic. Subscriptions, requests for back issues and changes of subscribers address should be sent to MSP.

Algebra \& Number Theory (ISSN 1944-7833 electronic, 1937-0652 printed) at Mathematical Sciences Publishers, 798 Evans Hall \#3840, c/o University of California, Berkeley, CA 94720-3840 is published continuously online. Periodical rate postage paid at Berkeley, CA 94704, and additional mailing offices.

ANT peer review and production are managed by EditFLow ${ }^{\circledR}$ from MSP.

\section{PUBLISHED BY}

- mathematical sciences publishers

nonprofit scientific publishing

http://msp.org/

() 2016 Mathematical Sciences Publishers 


\section{Algebra \& Number Theory}

Volume $10 \quad$ No. $10 \quad 2016$

Weight functions on Berkovich curves

MATTHEW BAKER and JOHANNES NICAISE

Nonvanishing of Dirichlet $L$-functions

2081

RIZWANUR KHAN and HIEU T. NGO

2093

Every integer greater than 454 is the sum of at most seven positive cubes

SAMIR SIKSEK

BERNARD LE STUM

JAN STEFFEn MÜlLER and Michael StOLL

BRUNO CHIARELLOTTO and CHRISTOPHER LAZDA

The Voronoi formula and double Dirichlet series

EREN MEHMET KiraL and FAN ZHOU

Pavel Etingof and Chelsea WALton 